\title{
TETHER FORCE CONSTRAINTS IN STOKES FLOW BY THE IMMERSED BOUNDARY METHOD ON A PERIODIC DOMAIN*
}

\author{
JOSEPH M. TERAN ${ }^{\dagger}$ AND CHARLES S. PESKIN ${ }^{\ddagger}$
}

\begin{abstract}
The immersed boundary method is an algorithm for simulating the interaction of immersed elastic bodies or boundaries with a viscous incompressible fluid. The immersed elastic material is represented in the fluid equations by a system or field of applied forces. The particular case of Stokes flow with applied forces on a periodic domain involves two related mathematical complications. One of these is that an arbitrary constant vector may be added to the fluid velocity, and the other is the constraint that the integral of the applied force must be zero. Typically, forces defined on a freely floating elastic immersed boundary or body satisfy this constraint, but there are many important classes of forces that do not. For example, the so-called tether forces that are used to prescribe the simulated configuration of an immersed boundary, possibly in a time-dependent manner, typically do not sum to zero. Another type of force that does not have zero integral is a uniform force density that may be used to simulate an overall pressure gradient driving flow through a system. We present a method for periodic Stokes flow that when used with tether points, admits the use of all forces irrespective of their integral over the domain. A byproduct of this method is that the additive constant velocity associated with periodic Stokes flow is uniquely determined. Indeed, the additive constant is chosen at each time step so that the sum of the tether forces balances the sum of any other forces that may be applied.
\end{abstract}

Key words. Stokes equations, immersed boundary method, numerical methods

AMS subject classifications. 65M06, 65, 76F07, 74F 10

DOI. $10.1137 / 080720217$

1. Introduction. Interactions between incompressible viscous fluids and immersed structures are ubiquitous in problems arising in physics, engineering, and biology. Flow past a cylinder is a typical model problem for separated flows and boundary layer theory; suspensions of flexible fibers and filaments can give rise to non-Newtonian fluidic behavior [13], [24], [27]; micro-organisms often utilize flexible flagella for locomotion [3]. Peristaltic pumping, a common mechanism in digestion and in the biofluids of reproduction, is driven by propagating a contractile wave along an elastic-fluid boundary [19]. The immersed boundary method is a general framework for such problems involving fluid-structure interaction. Although one might argue that flow past a fixed cylinder is not a problem of fluid-structure interaction, it should be noted that such problems can be solved by the immersed boundary method as if they were fluid-structure interaction problems. This is done by allowing the boundary of the cylinder to move but by tethering each point of the boundary to its target position by a stiff spring. Lai and Peskin used this technique for simulating flow past a cylinder in demonstrating the reduced numerical viscosity of their formally second order accurate immersed boundary method for the Navier-Stokes equations [12]. Beyer used such forces for modeling the cochlea with the immersed boundary method [2]. Goldstein, Handler, and Sirovich used a body force density to provide feedback in modeling flow around cylinders and in turbulent channels [9]. Iaccarino

${ }^{*}$ Received by the editors April 3, 2008; accepted for publication (in revised form) June 5, 2009; published electronically August 14, 2009.

http://www.siam.org/journals/sisc/31-5/72012.html

${ }^{\dagger}$ Department of Mathematics, UCLA, Los Angeles, CA 90095-1555 (jteran@math.ucla.edu).

${ }^{\ddagger}$ Department of Mathematics, Courant Institute of Mathematical Science, 251 Mercer St., New York, NY 10012-1110 (peskin@cims.nyu.edu).

3404 
and Verzicco later pointed out in their review article on such methods for turbulent flow that the method of Goldstein, Handler, and Sirovich is physically just a spring and damper model [10]. Saiki and Biringen [21] used high-order finite differences to suppress the numerical oscillations caused by the forcing term in [9] for flow past a cylinder, and Mohd-Yusof developed another forcing model with a less-stringent time step restriction [16]. This force-based technique for maintaining boundary conditions is central to the present paper.

The immersed boundary method is both a mathematical formulation and a numerical scheme incorporating a Lagrangian representation of immersed elastic structures and an Eulerian representation of the viscous incompressible fluid. These two representations are linked via Dirac delta functions. The Lagrangian immersed boundary applies a singular field of force to the Eulerian fluid and in turn moves at the local fluid velocity. Spatial discretization is based on a fixed Cartesian mesh for the fluid variables and a moving curvilinear mesh for the Lagrangian variables. A regularized approximation to the Dirac delta function is used to link the two sets of variables [18].

This paper is concerned with fluid-structure interaction problems in which the fluids are viscous enough that the inertia of the structure and the fluid can be neglected. In such cases, the inertial terms in the incompressible Navier-Stokes equations (including both $\rho \frac{\partial \mathbf{u}}{\partial t}$ and $\left.\rho \mathbf{u} \cdot \nabla \mathbf{u}\right)$ can be omitted yielding the incompressible Stokes equations. These equations are biharmonic. There are many numerical methods for fluid-structure interaction in this context. Immersed boundary based approaches can be found in [7], [6], [8], and [28]. The harmonic nature of the equations admits the use of Green's functions (Stokeslets) [20]. Cortez, Fauci, and Medovikov combine an immersed boundary method with regularized Stokeslets [5]. A second order immersed interface approach to the problem is given by LeVeque, Li, and Ito in [14] and [15]. Newren et al. have recently come up with an unconditionally stable discretization of the problem [17]. More stability analysis was also done by Stockie and Wetton in [25] and by Tu and Peskin in [28].

The Stokes equations, when employed in conjunction with periodic boundary conditions, have the mathematical implications (1) that the fluid velocity is determined only up to an additive constant and (2) that the integral of the force per unit volume applied to the fluid over the whole domain must be zero [1]. The latter constraint is of particular significance in an immersed boundary formulation of the problem, given that the immersed structure appears in the fluid equations in terms of the force that it applies to the fluid, and there are cases in which it is not obvious that we want to live with the constraint that the sum of these forces must be zero. The nonuniqueness of the fluid velocity is also disturbing, since the average of the fluid velocity over the whole domain is often the quantity of greatest interest and since this seems to be precisely what we cannot determine! A simple approach to the problem is to simply subtract away the integral of the force over the domain; however, such an approach would make the average velocity undetermined resulting in unacceptable loss of information.

Typically, the forces generated by an immersed elastic boundary do integrate to zero, but there is one important class of forces that do not: the so-called tether forces. Tether forces may be used to drive prescribed motion of an immersed structure or of some part of such a structure. In approaches other than the immersed boundary method, the formulation of boundary conditions between the solid and the fluid typically involves changing the numerical stencil that appears in the equations for the Eulerian fluid variables, and this can complicate implementation when done along an 
irregular moving boundary. The immersed boundary method, however, easily incorporates boundary conditions between the fluid and the solid via the regularized Delta functions without any need to modify the numerical stencil of the fluid equations anywhere. Given this freedom, it is advantageous to use periodic boundary conditions because they imply the same numerical stencil everywhere, even at the boundaries of the domain, which are not really boundaries at all in the periodic case. A related advantage of periodic boundary conditions is that they allow for the use of the fast Fourier transform algorithm in solving the fluid equations. In the present work, there is one further advantage of periodic boundary conditions: We are interested in peristaltic pumping, in which a wave of contraction generates net flow in the direction of wave propagation. Such a problem can be nicely formulated on a periodic domain, where the period is chosen as the wavelength of the prescribed motion of the boundaries.

Another type of force that does not integrate to zero is a constant body force, which may be used to drive fluid through a domain. Such a force may be used in conjunction with tether forces to provide a load against which a peristaltic pump is working. We will show that when one uses tether forces together with any other applied external force in periodic Stokes flow, there is a unique choice of additive fluid velocity which ensures that the forces applied to the fluid overall will always integrate to zero.

2. Stokes equations with immersed boundary. We consider a viscous incompressible fluid in a periodic domain $\Omega$ containing an immersed massless boundary $\Gamma$, the configuration of which is denoted by $\mathbf{X}(\xi, t)$, where $\xi$ is a reference Lagrangian parameter. We assume that inertia is completely negligible so that the flow is governed by the incompressible Stokes equations:

$$
\begin{array}{r}
\mu \Delta \mathbf{u}=\nabla p-\mathbf{f}, \\
\nabla \cdot \mathbf{u}=0,
\end{array}
$$

where $\mathbf{u}$ is the fluid velocity, $p$ is the fluid pressure, $\mathbf{f}$ is the force per unit volume that is applied to the fluid, and $\mu$ is the constant fluid viscosity. It will be more useful numerically to rewrite these equations in terms of an intermediate velocity $\mathbf{u}^{*}$ and scalar $p^{*}$ as

$$
\begin{array}{r}
\mu \Delta \mathbf{u}^{*}=-\mathbf{f}, \\
\Delta p^{*}=\nabla \cdot \mathbf{u}^{*}, \\
\mathbf{u}=\mathbf{u}^{*}-\nabla p^{*},
\end{array}
$$

where the pressure $p$ is related to $p^{*}$ as $p=-\mu \Delta p^{*}$. This decomposition is similar to the projection methods used for the Navier-Stokes equations [4] and requires commuting $\Delta$ and $\nabla$ operators. For Stokes flow, the above decomposition is exact. A similar decomposition can be done by first computing the pressure from the applied force $(\Delta p=\nabla \cdot \mathbf{f})$ as was done in [8]. We have found, however, that it is a more stable numerical procedure to compute an intermediate velocity first, as above, and only after that to perform a projection.

The immersed boundary method links the fluid and solid variables by applying a field of force per unit volume $\mathbf{f}_{\mathrm{IB}}(\mathbf{x}, t)$ to the fluid. This Eulerian force density is derived from its Lagrangian counterpart $\mathbf{F}_{\mathrm{IB}}(\xi, t)$ via the Dirac delta function. The Dirac delta function is used again to evaluate the Eulerian fluid velocity $\mathbf{u}(\mathbf{x}, t)$ at 
the material points $\mathbf{x}=\mathbf{X}(\xi, t)$ that define the spatial configuration of the immersed boundary. The result is the following system of equations:

$$
\begin{array}{r}
\mu \triangle \mathbf{u}=\nabla p-\mathbf{f}_{\mathrm{IB}}-\mathbf{f}_{\mathrm{AP}}, \\
\nabla \cdot \mathbf{u}=0, \\
\mathbf{f}_{\mathrm{IB}}(\mathbf{x}, t)=\int_{\Gamma} \mathbf{F}_{\mathrm{IB}}(\xi, t) \delta(\mathbf{x}-\mathbf{X}(\xi, t)) d \xi \\
\frac{\partial \mathbf{X}}{\partial t}(\xi, t)=\int_{\Omega} \mathbf{u}(\mathbf{x}, t) \delta(\mathbf{x}-\mathbf{X}(\xi, t)) d \mathbf{x} .
\end{array}
$$

Here, $\mathbf{f}_{\mathrm{AP}}(\mathbf{x}, t)$ is an externally applied force per unit volume. Note that the integration of (2.1) over the periodic domain $\Omega$ yields the constraint that the sum of all externally applied and immersed boundary based forces integrate to zero over $\Omega$ :

$$
\int_{\Omega}\left(\mathbf{f}_{\mathrm{IB}}+\mathbf{f}_{\mathrm{AP}}\right) d \mathbf{x}=\mathbf{0}
$$

It should also be noted that solutions of the Stokes equations on a periodic domain are only unique up to an additive constant velocity $\mathbf{u}_{\mathrm{c}}$ at each instant of time. Such solutions can be written as $\mathbf{u}(\mathbf{x}, t)=\hat{\mathbf{u}}(\mathbf{x}, t)+\mathbf{u}_{\mathrm{c}}(t)$, where $\int_{\Omega} \hat{\mathbf{u}}(\mathbf{x}, t) d \mathbf{x}=0$ and where $\mathbf{u}_{\mathrm{c}}(t)$ is independent of $\mathbf{x}$.

2.1. Tether forces. When the motion of any portion of the Lagrangian immersed boundary is known, we denote it by $\mathbf{Z}(\xi, t)$ and create tether forces of the form

$$
\mathbf{F}(\xi, t)=-k(\mathbf{X}(\xi, t)-\mathbf{Z}(\xi, t)),
$$

where $k$ is a large spring constant. These forces keep the relevant part of the immersed boundary close to its prescribed configuration $\mathbf{Z}$.

A common circumstance in applications of the immersed boundary method is that the forces applied by the immersed boundary to the fluid are derived from an elastic energy function that is invariant under translation, in which case the integral of the immersed boundary force is guaranteed to be zero. This is not the case when tether forces are used, however, since the prescribed configuration $\mathbf{Z}(\xi, t)$ destroys translation invariance. Therefore, the integral of the forces given by (2.11) cannot be expected to be zero.

3. Force integral constraint. Although tether forces do not typically integrate to zero, they can be used to satisfy the constraint that the integral of the total force on the fluid must be zero. In the process, the additive spatially constant velocity $\mathbf{u}_{\mathrm{c}}(t)$ introduced above is determined. To ensure that the forces integrate to zero, we require that they initially integrate to zero and also that the time derivative of their integral is zero. Before evaluating this time derivative, we make the assumption that the immersed boundary force $\mathbf{F}_{\text {IB }}$ may be decomposed into a sum of two terms

$$
\mathbf{F}_{\mathrm{IB}}=\mathbf{F}_{\mathrm{IB}, \mathrm{T}}+\mathbf{F}_{\mathrm{IB}, \mathrm{NT}},
$$

where $\mathrm{T}$ denotes the tether forces given by (2.11) and where NT denotes other, nontether immersed boundary forces, which satisfy

$$
\int_{\Gamma} \mathbf{F}_{\mathrm{IB}, \mathrm{NT}}(\xi, t) d \xi=\mathbf{0}
$$

Copyright $@$ by SIAM. Unauthorized reproduction of this article is prohibited. 
This condition will be satisfied, for example, as a consequence of a translation invariant energy-based conception of an elastic force and is the case for most nontether based elastic forces. Now (2.8) implies

$$
\int_{\Omega} \mathbf{f}_{\mathrm{IB}}(\mathbf{x}, t) d \mathbf{x}=\int_{\Gamma} \mathbf{F}_{\mathrm{IB}}(\xi, t) d \xi=\int_{\Gamma} \mathbf{F}_{\mathrm{IB}, \mathrm{T}}(\xi, t) d \xi .
$$

Therefore, the condition that the time derivative of the integral of the total force on the fluid must be zero gives

$$
\begin{aligned}
\mathbf{0} & =\frac{\partial}{\partial t}\left(\int_{\Omega} \mathbf{f}_{\mathrm{IB}}(\mathbf{x}, t) d \mathbf{x}+\int_{\Omega} \mathbf{f}_{\mathrm{AP}}(\mathbf{x}, t) d \mathbf{x}\right) \\
& =\frac{\partial}{\partial t}\left(\int_{\Gamma} \mathbf{F}_{\mathrm{IB}, \mathrm{T}}(\xi, t) d \xi+\int_{\Omega} \mathbf{f}_{\mathrm{AP}}(\mathbf{x}, t) d \mathbf{x}\right) \\
& =\int_{\Gamma}-k\left(\frac{\partial \mathbf{X}}{\partial t}-\frac{\partial \mathbf{Z}}{\partial t}\right) d \xi+\int_{\Omega} \frac{\partial \mathbf{f}_{\mathrm{AP}}}{\partial t} d \mathbf{x} .
\end{aligned}
$$

Since $\mathbf{u}=\hat{\mathbf{u}}+\mathbf{u}_{\mathrm{c}}$ and since $\mathbf{u}_{\mathrm{c}}$ is independent of $\mathbf{x},(2.9)$ reduces to

$$
\frac{\partial \mathbf{X}}{\partial t}(\xi, t)=\hat{\mathbf{U}}(\xi, t)+\mathbf{u}_{\mathrm{c}}(t)
$$

where

$$
\hat{\mathbf{U}}(\xi, t)=\int_{\Omega} \hat{\mathbf{u}}(\mathbf{x}, t) \delta(\mathbf{x}-\mathbf{X}(\xi, t)) d \mathbf{x} .
$$

Therefore, the condition that the time derivative of the integral of the force on the fluid must be zero reduces to the following:

$$
\mathbf{0}=-k \int_{\Gamma}\left(\hat{\mathbf{U}}(\xi, t)+\mathbf{u}_{\mathrm{c}}(t)-\frac{\partial \mathbf{Z}}{\partial t}(\xi, t) \partial t\right) d \xi+\int_{\Omega} \frac{\partial \mathbf{f}_{\mathrm{AP}}}{\partial t} d \mathbf{x} .
$$

This equation may be solved for $\mathbf{u}_{\mathrm{c}}(t)$ (which factors out of the integral because it is independent of $\xi$ ):

$$
\mathbf{u}_{\mathrm{c}}(t)=\frac{1}{k \int_{\Gamma} d \xi}\left[\int_{\Gamma}-k\left(\hat{\mathbf{U}}-\frac{\partial \mathbf{Z}}{\partial t}\right) d \xi+\int_{\Omega} \frac{\partial \mathbf{f}_{\mathrm{AP}}}{\partial t} d \mathbf{x}\right] .
$$

Thus, $\mathbf{u}_{\mathrm{c}}(t)$ is uniquely determined. (Recall that $\hat{\mathbf{u}}$ was uniquely determined by the condition that its integral over the whole periodic domain $\Omega$ should be zero. Although this normalization is convenient, it was not used earlier. In fact, an arbitrary function of time may be added to $\hat{\mathbf{u}}(\mathbf{x}, t)$ and hence to $\hat{\mathbf{U}}(t)$, in which case it is easy to see from the above formula for $\mathbf{u}_{\mathrm{c}}(t)$ that it will change in a compensatory manner. Thus, even if we do not specify a particular normalization for $\hat{\mathbf{u}}$, we still arrive at the result that the total velocity field is uniquely determined by the condition that the time derivative of the integral of the force on the fluid should be zero.)

4. Temporal discretization. The temporal approximation to (3.8) for the additive constant velocity $\mathbf{u}_{c}$ can be chosen carefully to ensure that the total applied forces at each time step integrate to zero exactly whenever a Stokes solve is performed. Denoting the time $n$ velocity by $\mathbf{u}^{n}(\mathbf{x})=\hat{\mathbf{u}}^{n}(\mathbf{x})+\mathbf{u}_{c}^{n}$, we first solve for the zero average velocity component of the flow $\hat{\mathbf{u}}^{n}$ assuming that the forces acting on the fluid 
integrate to zero. We then update the immersed boundary to $\hat{\mathbf{X}}^{n+1}$ and determine the average velocity $\mathbf{u}_{c}^{n}$ that will be required to correct the immersed boundary configuration in order to ensure that the forces integrate to zero at the beginning of the next time step:

$$
\begin{array}{r}
\mu \triangle \hat{\mathbf{u}}^{n}=\nabla p^{n}-\mathbf{f}_{\mathrm{IB}}^{n}-\mathbf{f}_{\mathrm{AP}}^{n}, \\
\nabla \cdot \hat{\mathbf{u}}^{n}=0, \\
\hat{\mathbf{X}}^{n+1}=\mathbf{X}^{n}+\Delta t \int_{\Omega} \hat{\mathbf{u}}^{n} \delta\left(\mathbf{x}-\mathbf{X}^{n}\right) d \mathbf{x}, \\
\mathbf{u}_{c}^{n}=\frac{1}{k \int_{\Gamma} d \xi}\left(k \int_{\Gamma} \frac{\mathbf{Z}^{n+1}-\hat{\mathbf{X}}^{n+1}}{\Delta t} d \xi+\frac{1}{\Delta t} \int_{\Omega} \mathbf{f}_{\mathrm{AP}}^{n+1} d \mathbf{x}\right), \\
\mathbf{X}^{n+1}=\hat{\mathbf{X}}^{n+1}+\Delta t \mathbf{u}_{c}^{n} .
\end{array}
$$

It is easy to check that the above choice of $\mathbf{u}_{\mathrm{c}}^{n}$ makes $\int_{\Omega} \mathbf{f}_{\mathrm{IB}}^{n+1}+\mathbf{f}_{\mathrm{AP}}^{n+1} d \mathbf{x}=\mathbf{0}$. That is, $\mathbf{u}_{\mathrm{c}}^{n}$ is the unique constant velocity that if used as in the final equation above results in forces at the beginning of the next time step that integrate to zero. We now show that this choice is consistent with (3.8) that was derived above in the time continuous case. To begin, note that

$$
\int_{\Omega} \mathbf{f}_{\mathrm{AP}}^{n} d \mathbf{x}=-\int_{\Gamma} \mathbf{F}_{\mathrm{IB}}^{n} d \xi=\int_{\Gamma} k\left(\mathbf{X}^{n}-\mathbf{Z}^{n}\right) d \xi
$$

and by this choice of $\mathbf{u}_{c}^{n}$

$$
\int_{\Omega} \mathbf{f}_{\mathrm{AP}}^{n+1} d \mathbf{x}=\int_{\Gamma} k\left(\mathbf{X}^{n+1}-\mathbf{Z}^{n+1}\right) d \xi
$$

Subtracting the two equations and dividing by $\Delta t$ shows that

$$
\int_{\Gamma} \frac{\mathbf{f}_{\mathrm{AP}}^{n+1}-\mathbf{f}_{\mathrm{AP}}^{n}}{\Delta t} d \mathbf{x}=k\left[\int_{\Omega} \hat{\mathbf{u}}^{n} \delta\left(\mathbf{x}-\mathbf{X}^{n}\right) d \mathbf{x}+\mathbf{u}_{c}^{n} \int_{\Gamma} d \xi-\int_{\Gamma} \frac{\mathbf{Z}^{n+1}-\mathbf{Z}^{n}}{\Delta t} d \xi\right] .
$$

Therefore,

$$
\mathbf{u}_{c}^{n}=\frac{1}{\int_{\Gamma} d \xi}\left[\frac{1}{k} \int_{\Omega} \frac{\mathbf{f}_{\mathrm{AP}}^{n+1}-\mathbf{f}_{\mathrm{AP}}^{n}}{\Delta t} d \mathbf{x}+\int_{\Gamma} \frac{\mathbf{Z}^{n+1}-\mathbf{Z}^{n}}{\Delta t} d \xi-\int_{\Omega} \hat{\mathbf{u}}^{n} \delta\left(\mathbf{x}-\mathbf{X}^{n}\right) d \mathbf{x}\right]
$$

which can be seen to be a first order approximation to the continuous result in (3.8).

5. Spatial discretization. We use a MAC-grid-based approximate projection method to solve (2.3)-(2.5), but note that our algorithm admits the use of any spatial discretization (e.g., a spectral discretization). Denoting $L_{h}$ as the standard five and seven point stencil discrete Laplacians in two and three dimensions, respectively, and $\mathbf{D}_{0}$ as the spatial centered difference, our approximation is

$$
\begin{array}{r}
\mu L_{h} \hat{\mathbf{u}}^{*}=-\mathbf{f}_{\mathrm{IB}}^{n}-\mathbf{f}_{\mathrm{AP}}^{n}, \\
L_{h} p^{* n}=\mathbf{D}_{0} \cdot \hat{\mathbf{u}}^{*}, \\
\hat{\mathbf{u}}^{n}=\hat{\mathbf{u}}^{*}-\mathbf{D}_{0} p^{* n} .
\end{array}
$$

(5.1)-(5.3) amount to three linear systems in two dimensions, and four in three dimensions. The matrices in each of these systems are the same. Note that $L_{h} \neq \mathbf{D}_{0} \cdot \mathbf{D}_{0}$, 
so our scheme has the structure of an inexact projection method. We use incompleteCholesky preconditioned conjugate gradient to solve each linear system.

Although the equations are written in immersed boundary form, the delta functions $\delta(\mathbf{x})$ must be approximated by discrete regularized delta functions $\delta^{I B}$ defined on the Eulerian grid $\Omega$. There are many ways to construct these grid-based approximations, all of which should converge to $\delta$ as the grid is refined and satisfy certain moment and quadratic conditions [18]. For example, it is very important for the constant velocity calculation $\left(\mathbf{u}_{c}^{n}\right)$ that the discrete delta functions satisfy the property that summation (representing integration) of a numerically "spread" Lagrangian quantity over the Eulerian domain $\Omega$ be the same as the summation of that quantity over the Lagrangian domain $\Gamma$. Such conditions give rise to a family of candidate discrete delta functions, from which we choose a four point delta function $\delta_{4 h}^{I B}$. This function has a support of four mesh widths in each spatial direction and is defined in terms of the function $\phi_{4}^{I B}$ with $\delta_{4 h}^{I B}(\mathbf{x})=\frac{1}{h^{2}} \phi_{4}^{I B}\left(\frac{x}{h}\right) \phi_{4}^{I B}\left(\frac{y}{h}\right)$ where

$$
\phi_{4}^{I B}(x)=\left\{\begin{array}{cc}
\frac{1}{8}\left(3-2|x|+\sqrt{1+4|x|-4 x^{2}}\right), & |x| \in[0,1], \\
\frac{1}{8}\left(5-2|x|-\sqrt{-7+12|x|-4 x^{2}}\right), & |x| \in[1,2], \\
0, \quad|x|>2 &
\end{array}\right.
$$

We use the trapezoidal rule to approximate the integrals in (4.4) yielding

$$
\mathbf{u}_{c}^{n}=\frac{1}{\Delta t \Sigma_{l=1}^{N} \Delta \xi_{l}}\left[\sum_{l=1}^{N}\left(\mathbf{Z}_{l}^{n+1}-\hat{\mathbf{X}}_{l}^{n+1}\right) \Delta \xi_{l}-\frac{1}{k} h^{2} \sum_{i, j} \mathbf{f}_{i, j}^{n+1}\right]
$$

for the spatially and temporally discretized additive constant velocity. Here, $\Delta \xi_{l}$ is the spacing between the discrete reference parameter values associated with each tether point.

\section{Examples.}

6.1. Mixing. Solutions to the Stokes equations satisfy a reversibility principle. Solutions corresponding to equal and opposite applied forces $\pm \mathbf{f}$ are themselves equal and opposite. This principle was demonstrated in a series of famous experiments with highly viscous Newtonian fluids by Taylor. In the experiments, the fluid is mixed until initially placed markers are considerably distorted. The mixing motion is then reversed. The fluid responds by exactly reversing its flow, returning the markers to their original configuration. In the spirit of these experiments, we demonstrate the reversibility principle with our algorithm. Two cylindrical peg mixers are placed in a Stokesian fluid and sweep out a time varying mixing trajectory. The trajectories are then reversed, returning the fluid to its initial configuration. The prescribed motion of the pegs is driven by tether forces (see Figure 6.1).

6.2. Pumping. Simulated pumping of Stokesian fluids is needed in industrial design as well as in biofluids. The most important characteristic of a pump is the relationship between the flow rate produced by the pumping mechanism and the pressure difference or load against which the pump is working. To study this relationship in the context of a periodic domain we need to apply a body force representing the load. This brings up the central problem of the present paper that the integral of the sum of all forces acting on the fluid must be zero and also that the fluid velocity seems to be unique only up to an additive constant velocity. This would seem to make the 


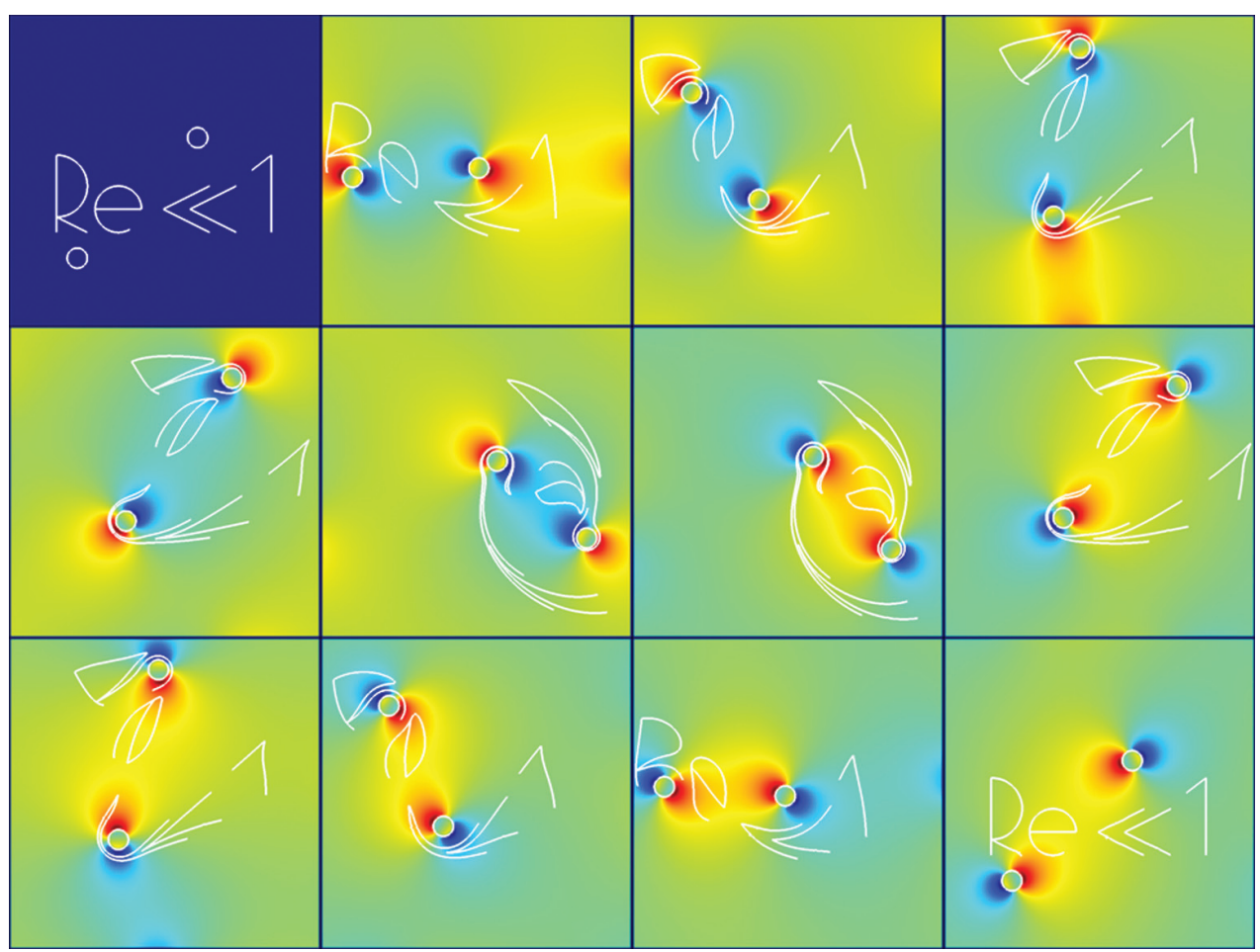

Fig. 6.1. Cylindrical pegs used to mix and then unmix a Stokesian fluid demonstrating the reversibility principle. The motion of the pegs is generated using tether forces. Color contours depict the fluid vorticity (note the change in sign of the vorticity upon reversal of mixing). Compare the last frame with the first, and note that even the sharp corners of the letters (represented here as passive, nonforce generating marker particles) are recovered.

computed flow rate undetermined. Our method admits the use of periodic boundary conditions, however, as long as tether forces are present in some portion of the domain, since these can then be used to balance any externally applied force in the manner described above. Specifically, the average velocity determined at each time step allows for accurate determination of the flow rate. In Figure 6.2 flow is driven through an irregular domain by a uniform body force (equivalent to a pressure gradient). In all other examples, $\mathbf{F}_{A P}=\mathbf{0}$. Tether forces are used to represent the irregular domain as immobile immersed Lagrangian boundaries. The tether forces allow for a nontrivial flow boundary without requiring any adjustment to the numerical stencil used in the difference scheme for the Stokes equations. Anisotropic domains like that shown in the figure have been used to rectify flow direction for complex viscous flows [23].

Peristalsis is a common pumping mechanism in the biofluids of digestion and reproduction. A peristaltic pump functions by propagating contractile waves along an elastic membrane enclosing the fluid. Material points in the membrane move primarily radially with phases chosen to generate a contractile wave that pumps fluid axially (see Figures 6.3 and 6.4). We model this mechanism with tether points $\mathbf{Z}(s, t)$ according to

$$
\mathbf{Z}(s, t)=\left(s, A \sin \left(\frac{2 \pi}{L}(s-\omega t)\right) \pm H\right)
$$




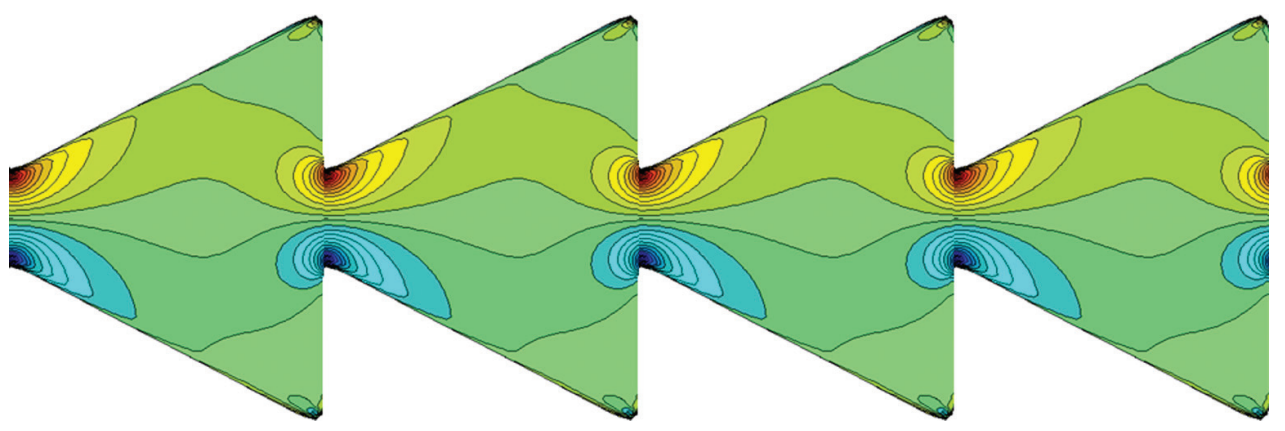

FiG. 6.2. Flow through an irregular domain simulated with stiff tether forces. Such domains can be used for flow rectification with complex viscous fluids (see, e.g., [23]). Resistance is the same in either direction for Stokes flows by the reversibility principle, but we have included this example to demonstrate the potential use of the method for examining such phenomena. The color contours show the vorticity of the fluid.

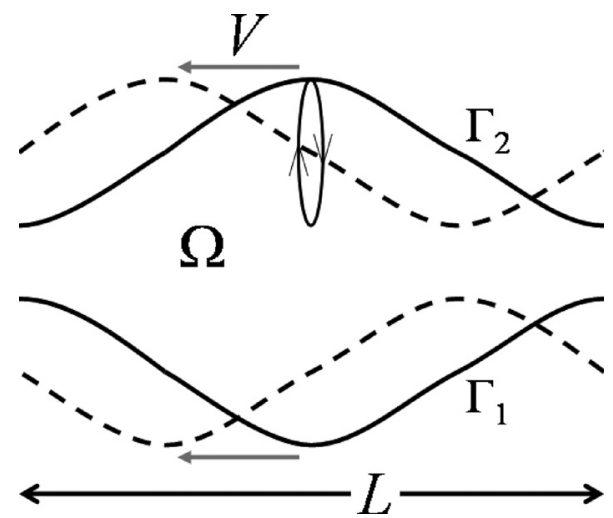

FIG. 6.3. Peristaltic pumps work by propagating a contractile wave along an elastic fluid boundary. Though the wave propagates in the direction of the bulk fluid flow, the motions of the material points in the boundary are primarily lateral. The figure depicts such a mechanism with $\Gamma_{1}$ and $\Gamma_{2}$ depicting the lower and upper elastic boundaries, respectively (see (6.1) for details).

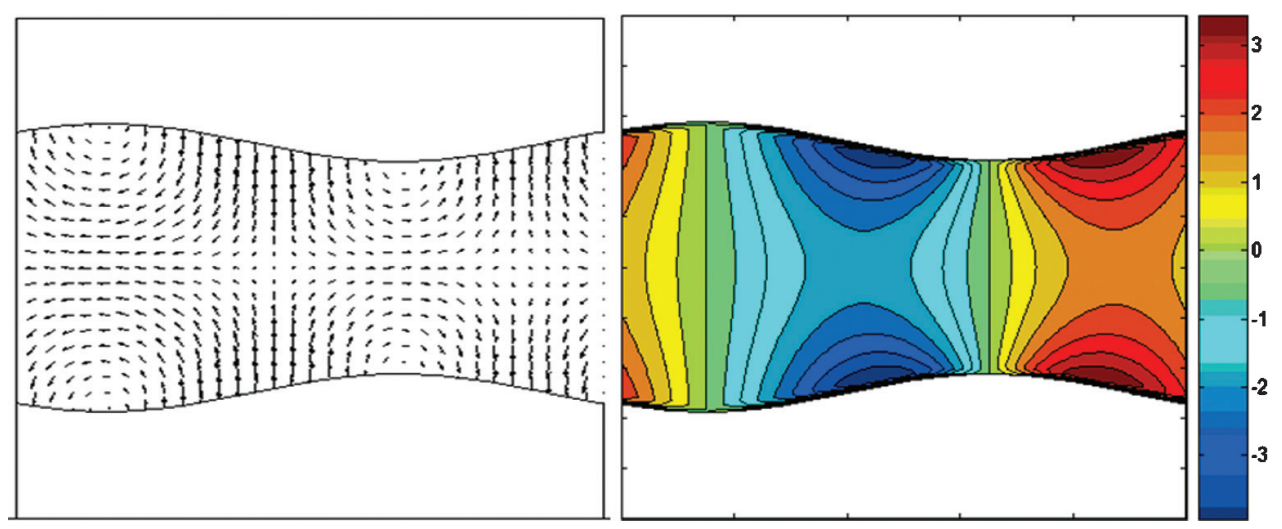

FIG. 6.4. The figure shows the velocity field and pressure contours in a peristaltic flow. The material points move up and down as the contractile wave moves from right to left. The net flow is also from right to left. 


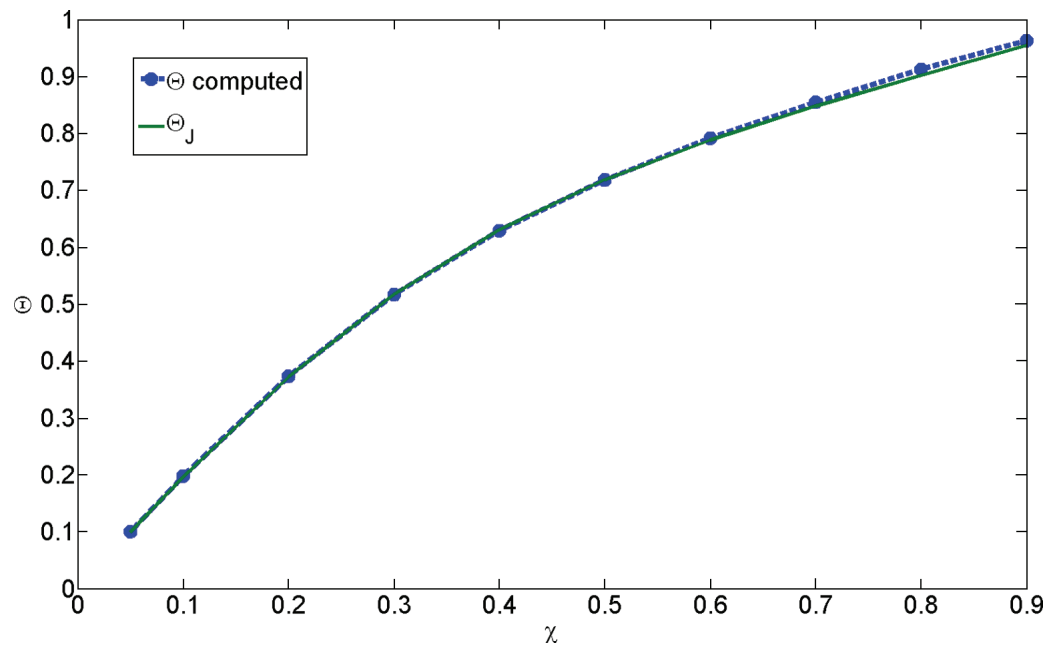

FIG. 6.5. The figure shows a comparison between the computed dimensionless mean flow rate $(\Theta)$ and the asymptotic approximation of Jaffrin and Shapiro [11]. The computed flow rate is in very good agreement with the asymptotic approximation for all values of the constriction ratio $\chi$.

The constriction ratio $\chi=\frac{A}{H}$ varies between 0 (boundary fixed in a straight, open configuration) and 1 (complete occlusion at the narrowest point) and sweeps out the range of possible flow domains (see Figure 6.5 for flow variation over a number of simulations using different values of $\chi$ ). For a Stokesian fluid, the flow induced by the pump increases monotonically with $\chi$. Our numerical approach allows us much more freedom to investigate all values of $\chi$. To quantify the effect of this parameter on the Stokes fluid we first define the mean flow velocity $Q$ as

$$
Q(s, t)=\int_{\Gamma_{1}(s, t)}^{\Gamma_{2}(s, t)} u(x(s), y) d y,
$$

where $\Gamma_{1}(s, t)$ describes the motion of the lower boundary and $\Gamma_{2}(s, t)$ describes the motion of the upper boundary of the tube. Also, the dimensionless mean flow rate $\Theta$ is defined as

$$
\Theta=\frac{1}{2 A c\left(T_{2}-T_{1}\right)} \int_{T_{1}}^{T_{2}} Q(s, t) d t
$$

where $T_{2}-T_{1}$ is some integer multiple of the pumping period $c=\frac{2 \pi \omega}{L}$. As defined above, $\Theta$ is a function of $s$, but in fact it has the same value for all $s$ because the motion of the boundary is that of a traveling wave and also because $T_{2}-T_{1}$ is a multiple of a period. The asymptotic analysis of [22] for a Stokes fluid showed that to zeroth order in a long-wavelength analysis the dimensionless mean flow rate is

$$
\Theta=\Theta_{S} \equiv \frac{3 \chi}{2+\chi^{2}}
$$

The expansion for the asymptotic analysis was done in the parameter $\alpha=\frac{2 \pi H}{L}$. Jaffrin and Shapiro [11] later improved upon this result by including second order effects in $\alpha$ to yield

$$
\Theta=\Theta_{J} \equiv \frac{15 \chi^{2}+2 \alpha^{2}\left[4\left(1-\chi^{2}\right)^{\frac{5}{2}}+\left(7 \chi^{2}-4\right)\left(1-\chi^{2}\right)\right]}{\chi\left[5\left(2+\chi^{2}\right)+6 \alpha^{2} \chi^{2}\left(1-\chi^{2}\right)\right]} .
$$


In Figure 6.5 we compare our computed dimensionless mean flow rate with $\Theta_{J}$ for varying $\chi$. The figure shows excellent agreement with Jaffrin's predicted values.

6.3. Locomotion. Taylor initially showed that a two-dimensional waving sheet could self-propel in a Stokesian fluid (despite the lack of inertia) [26]. Since then, much work has been done on the subject in relation to swimming microorganisms (as they typically interact with highly viscous fluids). Taylor started his analysis by assuming that the swimmer had a sinusoidal profile $\left(\mathbf{Z}_{c}\right)$ in the frame of its center of mass

$$
\mathbf{Z}_{c}(s, t)=(s, A \sin (k s-\omega t)) .
$$

That is, in the frame of reference of the swimmer's center of mass, the swimming motion is a simple sine wave (and hence has simple up and down motions of material points) propagating in the opposite direction to that of the locomotion. With this assumption, he showed that to leading order the waving sheet would move with velocity

$$
U=\frac{1}{2} k^{2} \omega^{2} A^{2}
$$

This is a common scenario for locomotion studies: Swimming kinematics $\left(\mathbf{Z}_{c}(s, t)\right)$ are hypothesized in the frame of the swimmer's center of mass, and the swimming speed $(U)$ induced by the given kinematics is then determined. These speeds can then be compared and contrasted to establish optimal swimming strategies, etc. for a given fluid. With such a framework, the fluid velocity in the frame of reference of the swimmer's center of mass at an infinite distance (measured orthogonal to the direction of locomotion) from the swimmer is opposite to the swimmer velocity. As the boundary of periodicity for the Eulerian domain gets larger, the constant velocity $\mathbf{u}_{c}^{n}$ determined by our method approaches this speed (we found that using a periodicity length of 1 and a swimmer amplitude of .025 and below was sufficient). Therefore, if tether forces are used to make a simulated swimmer adhere to the prescribed kinematics in the frame of the center of mass, the constant velocity determined by our method is exactly the negative of the swimmer's speed in the fluid. Figure 6.6 shows that our method can be used to accurately predict the swimming sheet velocity when compared with Taylor's asymptotic derivation.
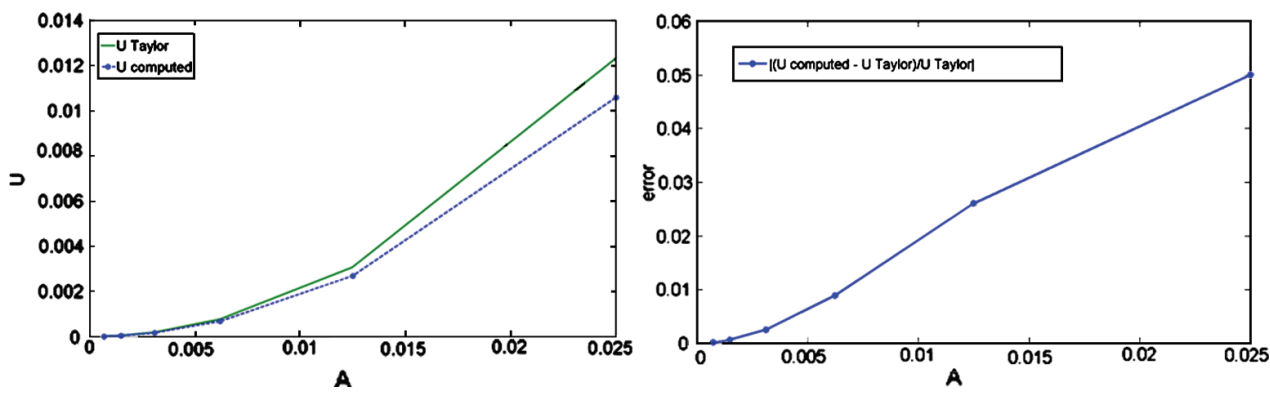

FIG. 6.6. Comparison of the computed Stokes swimming speed (U) with Taylor's asymptotic approximation for varying sinusoidal swimmer amplitudes (A). The plot on the left shows the speed variation with $A$. The plot on the right is of the relative error: $\frac{\left|U_{\text {computed }}-U_{\text {Taylor }}\right|}{U_{\text {Taylor }}}$. The agreement is very good for small values of $A$ which is to be expected given that the asymptotic approximation was done assuming small amplitude. The discrepancy for larger values of $A$ is thus not necessarily due to numerical error and may be due to errors in the asymptotic approximation. 
6.4. Simulation details. The method is explicit in time and first order in both space and time. All simulations were run with $\Delta x=\frac{1}{256}$ and with a tether stiffness of $k=600$. The explicit treatment of the temporal derivatives results in strict time step restrictions proportionate to $\Delta x^{2}$, and we found that $\Delta t=\frac{1}{5 k} \Delta x^{2}$ was sufficient for the examples considered. The favorable comparisons with asymptotics for both peristalsis and swimmer examples suggest that this choice of stiffness and resolution yielded nearly converged results.

7. Conclusion. In the present work, we have developed a method of solving problems involving the interaction of a highly viscous, Stokesian fluid with an immersed elastic boundary on a periodic domain. Periodic boundary conditions for a Stokes fluid require that the applied force on the fluid (including that from the immersed boundary method) integrate to zero over the domain. Our method satisfies this constraint with the aid of the often used tether force class of constraints. As a byproduct of the method, the free constant velocity associated with the periodic boundary conditions is uniquely determined. Periodic boundary conditions are especially desirable in the context of the immersed boundary method because they avoid introducing unwanted restrictions of the flow and allow the use of the same numerical stencil throughout the domain for all equations involving fluid variables, which improves the computational efficiency and admits the use of spectral methods. The efficacy of the method for simulating problems involving pumping and locomotion are demonstrated, and results are compared with asymptotic approximations. Finally, although this method is designed for Stokes flow, numerical methods for the Navier-Stokes equations may experience related problems with very low Reynolds numbers. For example, the linear equations for the velocities may become severely illconditioned with decreasing Reynolds number. A similar approach to that presented might be used to help prevent this ill conditioning.

\section{REFERENCES}

[1] G. K. BAtchelor, An Introduction to Fluid Dynamics, Cambridge University Press, Cambridge, 1967.

[2] R. P. Beyer, A Computational Model of the Cochlea Using the Immersed Boundary Method, Ph.D. thesis, University of Washington, Seattle, WA, 1989.

[3] S. Childress, Mechanics of Swimming and Flying, Cambridge Stud. Math. Biol., Cambridge University Press, Cambridge, 1981.

[4] A. J. Chorin, Numerical solution of the Navier-Stokes equations, Math. Comp., 22 (1968), pp. $745-762$.

[5] A. R. Contez, L. Fauci, And A. Medovikov, The method of regularized stokeslets in three dimensions: Analysis, validation and application to helical swimming, Phys. Fluids, 17 (2005), 031504.

[6] L. FauCI, Peristaltic pumping of solid particles, Comput. and Fluids, 21 (1992), pp. 583-598.

[7] L. Faudi And C. Peskin, A computational model of aquatic animal locomotion, J. Comput. Phys., 77 (1988), pp. 85-108.

[8] A. Fogelson And C. Peskin, A fast numerical method for solving the three-dimensional Stokes equations in the presence of suspended particles, J. Comput. Phys., 79 (1988), pp. 50-69.

[9] D. Goldstein, R. Handler, And L. Sirovich, Modeling a no-slip boundary with an external force field, J. Comput. Phys., 105 (1993), pp. 546-366.

[10] G. IACCARINO AND R. VERZICCO, Immersed boundary technique for turbulent flow simulations, Appl. Mech. Rev., 56 (2003), pp. 331-347.

[11] M. Y. Jaffrin and A. H. Shapiro, Peristaltic pumping, Annu. Rev. Fluid Mech., 3 (1971), pp. $13-37$.

[12] M. Lai And C. Peskin, An immersed boundary method with formal second order accuracy, J. Comput. Phys., 160 (2000), pp. 705-719.

[13] R. G. Larson, The Structure and Rheology of Complex Fluids, Oxford University Press, New York, 1998. 
[14] R. J. LeVeque and Z. LI, Immersed interface methods for Stokes flow with elastic boundaries or surface tension, SIAM J. Sci. Comput., 18 (1997), pp. 709-735.

[15] Z. Li And K. Iто, The Immersed Interface Method: Numerical Solutions of PDEs Involving Interfaces and Irregular Domains, Frontiers Appl. Math. 33, SIAM, Philadelphia, 2006.

[16] J. Mohd-Yusof, Combined Immersed Boundary/B-Spline Methods for the Simulations of Flow in Complex Geometries, Annual Research Briefs, Center for Turbulence Research, 1997, pp. 317-328.

[17] E. P. Newren, A. L. Fogelson, R. D. GuY, And R. M. Kirby, Unconditionally stable discretizations of the immersed boundary equations, J. Comput. Phys., 222 (2007), pp. $702-719$.

[18] C. S. Peskin, The immersed boundary method, Acta Numer., 11 (2002), pp. 479-517.

[19] C. Pozrikidis, A study of peristaltic flow, J. Fluid Mech., 180 (1987), pp. 515-527.

[20] C. PozRikidis, Boundary Integral and Singularity Methods for Linearized and Viscous Flow, Cambridge University Press, Cambridge, 1992.

[21] E. M. Saiki And S. Biringen, Numerical simulation of a cylinder in uniform flow: Application of a virtual boundary method, J. Comput. Phys., 123 (1995), pp. 450-465.

[22] A. H. Shapiro, M. Y. Jaffrin, and S. L. Weinberg, Peristaltic pumping with long wavelengths at low reynolds number, J. Fluid Mech., 37 (1969), pp. 799-825.

[23] T. M. Squires And S. R. Quake, Microfluidics: Fluid physics at the nanoliter scale, Rev. Modern Phys., 7 (2005), pp. 977-1026.

[24] J. M. Stockie and S. I. Green, Simulating the motion of flexible pulp fibres using the immersed boundary method, J. Comput. Phys., 147 (1998), pp. 147-165.

[25] J. M. Stockie And B. T. R. Wetton, Stability analysis for the immersed fiber problem, SIAM J. Appl. Math., 55 (1995), pp. 1577-1591.

[26] G. I. TAYLOR, Analysis of the swimming of microscopic organisms, Proc. Roy. Soc. Lond. Ser. A, 209 (1951), pp. 447-461.

[27] A. K. Tornberg And M. J. Shelley, Simulating the dynamics and interactions of flexible fibers in Stokes flows, J. Comput. Phys., 196 (2004), pp. 8-40.

[28] C. TU AND C. S. Peskin, Stability and instability in the computation of flows with moving immersed boundaries: A comparison of three methods, SIAM J. Sci. Stat. Comput., 13 (1992), pp. 1361-1376.

Copyright (c) by SIAM. Unauthorized reproduction of this article is prohibited. 